\title{
Effectiveness of Occupational Training Through Videoconferencing: Comparison with Classroom Training and Individual Differences
}

\section{Efectividad de la Formación Ocupacional Mediante Videoconferencia: Comparación con la Formación en el Aula y Diferencias Individuales}

\author{
Mar Iglesias and Jesús F. Salgado \\ University of Santiago de Compostela
}

\begin{abstract}
This article reports on a research in which a comparison is made between the effectiveness of classroom training and training through videoconference. The sample consisted of 561 adults that participated in training courses for entrepreneurs in both training modalities. The results were analyzed according to the first-level of effectiveness in Kirkpatrick's model of evaluation of training effectiveness. The results show that both training modalities were very well appraised by the participants, but there are differences in the instructor appraisal, who resulted slightly better assessed in the case of classroom training. Finally, the implications of the results are discussed and new research and potential moderators of effectiveness are suggested.

Keywords: effectiveness, classroom training, individual differences, videoconference.

Resumen. Este artículo incluye una investigación en la que se compara la eficacia de la formación en el aula y de la formación mediante videoconferencia. La muestra fueron 561 adultos que participaron en cursos formativos para emprendedores en ambas modalidades formativas. Los resultados se analizaron de acuerdo a la efectividad según el primer nivel del modelo de Kirkpatrick. Los resultados indican que ambas modalidades formativas fueron muy bien evaluadas por los participantes, pero existen diferencias en la valoración del monitor, que resultó ligeramente más favorable en el caso de la formación en el aula. Finalmente se discuten los resultados y sus implicaciones para la formación y se sugieren nuevas investigaciones y posibles moderadores de la eficacia.

Palabras clave: eficacia, formación en el aula, videoconferencia, diferencias individuales.
\end{abstract}

Training is the typical mechanism of increasing intellectual capital of organizations through the improvement of their human capital (Boudreau \& Ramstad, 2005). In this way, both organizations and employees benefit of training process: organizations benefit because they can count on personnel with better competencies to carry out their job functions and tasks, and employees benefit because the improved competencies acquired by training can provide added value of their professional profile and subsequently, can give them the access to better compensation packages, jobs, promotions, and so on (Salas, Tannenbaum, Kraiger, \& Smith-Jentsch, 2012).

Both public and private organizations invest important amounts of economic resources in employee train-

The authors want to acknowledge their gratitude to the management and personnel of IGAPE (Xunta de Galicia) for the support and the access to data.

Correspondence on this article should be sent to Mar Iglesias, Faculty of Labor Relations, University of Santiago de Compostela, Campus Vida, 15782, Santiago de Compostela. E-mail: mdelmar.iglesias@usc.es ing. Furthermore, employees invest their own economic and productive resources in their personal training. To give a couple of examples, it is estimated that organizations invested over $10,000 €$ billions in the USA (Patel, 2010), and in Spain the government invested over $3000 €$ in continuous education (to which the investment of private organizations and employees must be added).

Among other factors, investment is made in training because it is profitable for organizations. Individual studies, as well meta-analyses, have demonstrated that training has positive results on learning and knowledge acquisition (measured with both objective and subjective criteria), on individual performance, and on productivity. For example, Arthur, Bennett, Edens, and Bell, (2003) found an effect size of $d=.62$ for global effectiveness, $d=.63$ for learning criteria, and $d=.62$ for job performance. Salas, Nichols, and Driskell (2007) and Salas et al. (2008) found similar results.

Nevertheless, in times of economic crisis and increased competitiveness between organizations, the need to cut costs imposed on organizations means that 
investment in training is one of the first processes affected by budget cuts. For this reason, and for reasons of effectiveness, both organizations and employees have approached Information and Communication Technologies (ITCs) as a source of cheaper training programs. It is clear that ITCs offer large possibilities of reducing costs and extending the number of potential beneficiaries of training. For example, a number of the top universities (e.g., Harvard, MIT, Stanford) offer Internet-based courses and students from many countries are enrolled on these courses simultaneously. Two types of ITC-based training are becoming more and more common in recent years: on-line training (elearning) and distance training by videoconference.

With regards to on-line training (Web-based training), a recent meta-analysis carried out by Stizmann, Krager, Stewart, and Wisher (2006) found that on-line training showed similar results to classroom training, if the instruction mode and the content of training did not vary. These findings suggested that if training is well designed, it will be effective independently of the modality in which it is provided.

With regard to the effectiveness of videoconferencebased training, research is scarce (Kraiger, 2003; 2008). According to Noe (2002), this kind of training is characterized as being a type of instruction that allows for synchronic communication (as is the case of more the traditional training), and allows for real-time interaction between the instructor and the participants, as well as (less commonly) among the participants. Among its advantages are: (a) savings in travelling expenses and (b) possibility for employees and participants that are located in geographically dispersed places to receive the same instruction simultaneously, without the instructor having to visit each location. However, videoconferencing can also have disadvantages, among them the potential lack of interaction between the instructor and the audience or between participants. One of the positive traits of the traditional model of learning is that permits an significant amount of interaction between instructor and participants, which can contribute to a more positive learning climate, and an increased motivation of participants. This effect could be attenuated or even lost completely, in the case of videoconference-based training (Noe, 2002).

The advantages which have been mentioned (reduction of costs, increment of potential users, and simultaneous participation of employees in distant places without necessity of travelling) are clear, and do not require additional demonstration. However, one aspect that has not been examined enough is whether training effectiveness is the same in a traditional classroom environment and a distant environment which is mediated by technological advances, such as videoconference. Another point that has not yet been examined is whether or not users show differential reactions in presence or absence of a teacher or instructor in the training room.
Research on effectiveness of training is scarce at present and its objectives have varied greatly. In one of the first studies, Scott, Gururajan, Moloney, and Gururajan (2006) carried out a qualitative analysis of the videoconferencing, using the focus group technique. Their results demonstrated that users showed more preference for the use of videoconferences, ahead of electronic alternative systems, such as e-mail, chat or telephone. However, the generalizability of these results is limited due to the small sample size. In another study, Ferran and Watts (2008) examined the hypothesis that users (participants) suffer higher cognitive workload in the case of the videoconference in comparison with face-to-face training in the classroom. The incremented cognitive workload can make it necessary to use different processing systems in both training modalities. For instance, participants in a videoconference may give more attention to likeability of the instructor than to the quality of the material. The results of Ferran and Watts' (2008) study with medical professionals showed that participants were more influenced by likeability of the speaker than for the quality of the arguments, whereas the reaction was exactly the opposite one in the case of participants attending the same conference in the classroom.

Another of the few existing studies in this area was recently carried out by Dualde, Faus, Santonja, and Fernández-Lillmos (2009) in Spain. These researchers examined the effectiveness of a videoconference-based training course to implement pharmacy services. In a training course which included 13 videoconferences, Dualde et al. (2009) found that the program quality was evaluated as 'good' or 'very good' by the majority of the participants. Due to the nature of the design, Dualde et al.'s (2009) findings do not permit us to establish the differential effectiveness of the program in comparison with the results if the program were applied in the classroom.

This summary of the empirical studies already carried out shows up some important research limitations. For example, together with the differential effects on the cognitive workload suggested by Ferran and Watts (2008), differences might also exist with respect to the quality of the interpersonal relationships. From this perspective, the positive effects of the presence of the instructor and other participants could be reduced if training is by videoconference. For example, feedback process from instructor to participant and from participant to instructor could be negatively affected, with the subsequent loss of efficiency in the training process. Similarly, training motivation could be different in the case of videoconferencing, with extra effort required from instructors and teachers in order to maintain attention of participants.

For the reason outlined above, the present study aims to contribute to the literature examining the effectiveness of videoconferences. We report research in which the results of two modalities of training (class- 
room training and videoconference training) were compared in a large sample of adults.

The best-known and most widely-used model for the evaluation of the effectiveness of training programs is the model of four levels of Kirkpatrick (1994; see also Alliger \& Janak, 1988). According to Kirkpatrick, the evaluation of training programs should be made attending to the following four questions: (1) What is the reaction of participants? Do they appraise training? (2) What is learned? Do participants improve competencies (skills and knowledge) with the training program? (3) What are the effects on performance? Do participants change (improve) their behaviors and job performance? (4) What are the effects on productivity? Do changes of behavior and performance produce economic returns? In the present study, our examination will center on the first level of Kirkpatrick's model.

Based on the results of Stizmann et al. (2006) and of Chipps, Brysiewicz, \& Mars (2012), we conjecture that there are not relevant differences in the effects sizes between classroom training and videoconference-based training, and that if there are, the effect sizes will be of small or medium magnitude $(d=.20 \sim$ .50). We also suggest that no differences will be found between men and women.

\section{Method}

Participants and procedure. The sample consisted of 561 unemployed individuals, who were enrolled on training courses of entrepreneurship and self-employment. The courses were provided both in classroom training modality and in videoconference-based modality. 395 individuals participated in classroom training courses and 166 in videoconference-based courses. 257 women and 138 men participated in the classroom courses. All the participants were women in the videoconference-based courses. The size of groups ranged from 18 to 83 , with an average of 37 individuals per course, for both the classroom courses and the videoconference-based courses.

Participation was voluntary, being the only requirement that the individual had to be unemployed at the course time. The content course and the learning material were the same for the two training modalities. The course instructor was held constant in the two modalities with the objective of ensuring that if there were differences in effectiveness, these would be due to the training modality and not to other potential variables such as differences in the content, material, or instructor.

At the end of the course, participants evaluated the course results anonymously on a series of scales directed at assessing several aspects (see below). Due to the fact that the main objective of the course was to train participants as future entrepreneurs, the best indicators of training effectiveness in this case are the ratings of the participants.
The courses were given from October $20^{\text {th }}, 2011$ to July $28^{\text {th }}, 2012$. They were a part of the training programs developed by Instituto Gallego para la Promoción Económica (Galician Institute for Economic Promotion) of the Xunta de Galicia (Galician Government).

Training effectiveness. Eleven Likert-type scales were used to assess the effectiveness of the training course. The scale had a range of 1 to 10 , with the higher pole indicating better effectiveness. The participants assessed the following characteristics of the course: (1) interest and relevance, (2) climate, (3) quality, (4) room, (5) course information, (6) possibilities to participate during the course, (7) explanations of the instructor, (8) style of the instructor, (9) possibilities to interact with the instructor, (10) adequacy of the instructor for the course, and (11) competence level of the instructor. Furthermore, we created three compounds: one compound with the first three scales referring to the course, a second compound with the next three scales referring to the course organization, and a third compound with the last five scales referring to the instructor. The main reason for creating these three compounds is that they are psychometrically more reliable than the single scales.

\section{Results}

The mean and standard deviation of the variables used in the study to examine the effectiveness of the two training modalities appear in Table 1. As can be seen, the mean score is very high in all variables for both the classroom training modality and the conference-based training modality, ranging from 8.30 to 9.38 for the classroom modality and from 7.96 to 8.87 for the videoconference modality. It can therefore be concluded that the participants rated these two training modalities as very good. A second important result is that the instructor received the highest ratings in the two modalities of training. A third relevant result is that the ratings are slightly higher in the classroom modality than in the videoconference modality for the majority of the variables.

The results for the compounds of training effectiveness appear in Table 2. The first remarkable result for the compounds is that the reliability is very large in all three cases, with the reliability of the instructor compound, which showed an Alpha coefficient of .88, being especially high. With regard to the mean scores, the potential range of the results was from 3 to 30 for the compound of course and course organization and from 5 to 50 for the compound referring to the instructor. As can be seen, the results are very high in the three cases, just as with the single variables. In addition, the classroom modality of training received slightly better ratings than videoconference-based training. 
Table 1. Descriptive Statistics and Reliability of Variables of Training Effectiveness

\begin{tabular}{lcccc}
\hline & \multicolumn{2}{c}{ Classroom } & \multicolumn{2}{c}{ Videoconference } \\
\hline Variable & $\begin{array}{c}\text { M } \\
(\mathrm{n}=395)\end{array}$ & \multicolumn{2}{c}{$\begin{array}{c}\text { SD } \\
(\mathrm{n}=166)\end{array}$} \\
& & & & SD \\
& & & & \\
\hline Course & 8.44 & 1.54 & 8.02 & 1.69 \\
$\quad$ Interest & 8.60 & 1.42 & 8.50 & 1.54 \\
Climate & 8.30 & 1.67 & 7.96 & 1.81 \\
$\quad$ Quality & & & & \\
Organization & 8.61 & 1.45 & 8.70 & 1.45 \\
$\quad$ Room & 8.58 & 1.51 & 8.19 & 1.63 \\
Information & 8.55 & 1.54 & 8.24 & 1.75 \\
$\quad$ Participation & & & & \\
Monitor & 9.38 & 0.78 & 8.87 & 1.09 \\
$\quad$ Explanations & 9.30 & 0.83 & 8.82 & 1.22 \\
Style & 9.36 & 0.80 & 8.75 & 1.35 \\
Interaction Possibilities & 9.22 & 0.91 & 8.63 & 1.39 \\
Adequacy & 9.36 & 0.81 & 8.71 & 1.27 \\
$\quad$ Competence Level & & & & \\
\hline
\end{tabular}

Table 2. Descriptive Statistics and Reliability of the Compounds of Training Effectiveness

\begin{tabular}{lccccc}
\hline & \multicolumn{2}{c}{ Classroom } & \multicolumn{4}{c}{ Videoconference } \\
\hline Variable & $\mathrm{M}$ & $\mathrm{SD}$ & $\mathrm{M}$ & $\mathrm{SD}$ & $\alpha$ \\
\hline Course & 25.27 & 4.06 & 24.47 & 4.51 & .81 \\
Organization & 25.75 & 3.91 & 25.13 & 4.23 & .83 \\
Instructor & 46.63 & 3.20 & 43.87 & 5.26 & .88 \\
\hline \multicolumn{3}{c}{$(\mathrm{n}=395)$} & \multicolumn{4}{c}{$(\mathrm{n}=166)$} \\
\hline
\end{tabular}

Tables 3 and 4 contain the results of the statistical contrasts between the means of the classroom training and the videoconference training. These contrasts were carried out to test whether or not there are statistically significant differences in the ratings of the two training modalities. In the case of the single variables, climate and classroom do not show significant differences but there are significant differences for the rest of variables. The differences in the variables related to the instructor are especially relevant. In the case of the compounds, there are significant differences in two of them: the course compound and the trainer compound.

Nevertheless, the previous results do not offer all the information needed to assess the differential effectiveness of a training modality over the other. The existence of statistically significant differences means that they are real, but the contrasts do not inform as the magnitude of such differences. In the last column of Table 3 and 4 Cohen's $d$ coefficient can be found, which indicates the effect size, or magnitude, of the difference between the two training modalities. According to Cohen (1988, see also Murphy \& Myors, 1998) $d$ values of .20 indicate small effects, values $d$ of
Table 3. Results of the Comparisons between the Means of the Training Effectiveness Variables for Classroom Training and Videoconference

\begin{tabular}{lccc}
\hline Variable & $\mathrm{F}$ & $p$ & $d$ Cohen \\
\hline Course & & & \\
$\quad$ Interest & 8.13 & .005 & .26 \\
$\quad$ Climate & 0.59 & .443 & .07 \\
$\quad$ Quality & 4.56 & .033 & .20 \\
Organization & & & \\
$\quad$ Room & 0.41 & .522 & -.06 \\
$\quad$ Information & 7.49 & .006 & .25 \\
$\quad$ Participation & 3.43 & .065 & .19 \\
$\quad$ Instructor & & & \\
$\quad$ Explanations & 39.04 & .000 & .58 \\
$\quad$ Style & 29.02 & .000 & .50 \\
$\quad$ Interaction Possibilities & 43.93 & .000 & .61 \\
$\quad$ Adequacy & 35.46 & .000 & .55 \\
$\quad$ Competence Level & 53.80 & .000 & .67 \\
& & & \\
\hline
\end{tabular}

Note. D.F. $=1,557$

Table 4. Results of the Comparisons between the Means of the Training Effectiveness Compounds for Classroom-Training and Videoconference

\begin{tabular}{lrcc}
\hline Variable & $\mathrm{F}$ & $p$ & $d$ Cohen \\
\hline Course & 4.26 & .039 & .15 \\
Organization & 2.21 & .138 & .19 \\
Instructor & 57.71 & .000 & .70 \\
\hline
\end{tabular}

Note. D.F. $=1,557$

.50 indicate medium effects, and values $d$ of .80 are indicators of large effects. As Cohen's $d$ is a standardized index of the impact of treatment on the dependent variable, in our case it would be the effect of the training modality on the effectiveness variables. As $d$ is expressed in terms of standard deviation units, the comparison is possible of the effectiveness of a training modality with respect to the other. For instance, a $d$-value of .20 means that the difference between the training modalities is only $20 \%$ as large as the standard deviation of the measure of comparison that has been used (e.g., climate).

As can be seen in the last column of Tables 3 and 4, the effect sizes are very small or null in all the variables related with the course and with the organization of the course, and they are of medium size for the variables related with the instructor. Considered globally, the results for the compounds showed that the training modality was not very relevant for the course and for the course organization ( $d$ values of .15 and .19), but the differences should be taken into account with regard to the instructor $(d=.70)$.

The analysis of the differences between men and women in the ratings of the training effectiveness was only carried out with regard to the classroom modality because, as was mentioned above, all participants in the videoconference modality were women. The mean 
contrast of men and women reveals no differences in any variable between the two genders, which we interpret as confirmation that the effectiveness of training is no affected by gender differences in the case of classroom training.

\section{Discussion}

The importance of the training of human capital is an undisputed organizational necessity at present. Public and private organizations need human capital to be highly-qualified in order to have a competitive advantage. However, at the same time, training is an expensive process and it means the balancing of effectiveness and optimal level of cost. In this sense, organizations have opted in the last few years by training processes based on the possibilities arising from the use of ITCs, including on-line learning and videoconference-based training. However, as Kraiger (2003, 2008) has suggested, there are few studies that justify the adoption of ITC-based instruction, in which the effectiveness of training has been examined or costbenefit analyses or a utility analyses have been done (Cascio 1991, Salgado, 2007).

The main objective of this study was to contribute to research on the effectiveness of the training modalities, making a comparison of the differential effectiveness of classroom training and the videoconference-based training. This latter modality has been proposed as an important alternative, especially when the participants have difficulties to travel or the costs are prohibitively high. Furthermore, it permits the simultaneous access of a potentially large number of individuals because it is not limited to a single physical environment (classroom). These advantages seem clear, but before a generalization of the use of videoconference in training context, two complementary but independent aspects have to be examined. First, it must be confirmed whether the effectiveness of training is essentially the same or that the differences are relatively small in comparison with other training modalities. Second, it seems necessary to demonstrate that the change is justified through a cost-benefit analysis (or a utility analysis). The first aspect was the objective of the current study.

The results of this study suggest that the two modalities of training are highly effective, with average scores of 8 or higher (on a scale of 1 to 10). It can, therefore, be concluded that both modalities are valid and valuable options for the training programs. A second conclusion is that when the content is similar, the participant sees no significant differences in the fact that the content is explained in the classroom or through videoconferences.

However, the instructor-related variables were rated better for the classroom training than for the videoconference-based training. Even though the differences are not large, they are real. The magnitude was .70, which is around 1 point in a scale from 1 to 10 . This means that the differential effect of the instructor should be more exhaustively examined in future studies. In the present study, we had controlled the effect of the instructor, because it was constant in the various courses. However, it seems that the instructor's characteristics may be a key element in the effectiveness of videoconferences as a way of delivering training. In this research, the instructor was rated highly in both modalities, but it is possible that the rating might be different for the two modalities if the level of qualifications of the instructor was not so high.

An important difficulty of the instructors in the case of videoconference is the reduction of the opportunities for instructor interaction with the participants during the training time, breaks, and at the end of the course. In countries with collectivistic cultures such as Spain (Hofstede, Hofstede, \& Minkov, 2010), the interaction with the instructor during breaks and at the end of the sessions is especially well-valued and is very frequent. The limitation or the impossibility of such interactions in the case of the videoconference adds an important difficulty to the job of the instructor. For this reason, the design of videoconference-based training programs should consider the inclusion of substitutes of the interactions, and in particular, breaks that permit informal interactions between the trainer and the participants.

We have not examined whether the rating of effectiveness of each type of training modality is related with the education level of the participants. Additional studies should be devoted to this issue. It could be possible that the effectiveness of one modality was more related with a particular level of education. For example, videoconferences can be more effective with higher levels of education and the classroom training can be more effective with lower levels. The potential effects of age on the effectiveness ratings were not examined either. It is possible that the appraisal of the videoconference-based training is higher for younger people (because they are more familiar with ITCs) and that the appraisal of older people is higher for the classroom training. Future studies should investigate this issue.

In summary, the current study provides information about the potential effectiveness of videoconference as a substitute of classroom training. The results suggest that it can be a useful alternative modality for delivering training. However, additional studies are very much needed, especially cost-benefit analyses and studies of moderators of its effectiveness.

\section{References}

Alliger, G. \& Janak, E. A. (1989). Kirkpatrick's level of training criteria: Thirty years later. Personnel Psychology, 42, 331-342.

Arthur, W. Jr., Bennett, W. Jr., Edens, P. S., \& Bell, S. T. (2003). Effectiveness of training in organizations: A 
meta-analysis of the design and evaluation features. Journal of Applied Psychology, 88, 234-245.

Boudreau, J., \& Ramstad, P. (2005). Talentship, talent segmentation, and sustainability: A new HR decision science paradigm from a new strategy definition. Human Resource Management, 44, 129-136.

Cascio, W. F. (1991). Costing human resource: The financial impact of behavior in organizations. Boston, MT: PWS Kent.

Chipps, J., Brysiewicz, P., \& Mars, M. (2012). A systematic review of the effectiveness of video-conference-based tele-education for medical and nursing education. Worldviews Evidence Based Nursing, 9, 78-87.

Cohen, J. (1988). Statistical power analysis for the behavioral sciences ( $2^{\mathrm{a}}$ ed). Hillsdale, NJ: Erlbaum.

Dualde, E., Faus, M. J., Santonja, F. J., \& Fernández-Llimos, F. (2009). Effectiveness of a videoconference training course on implementing pharmacy services. Pharmacy World and Science, 31, 638-642.

Ferran, C. \& Watts, S. (2008). Videoconferencing in the field: A heuristic processing model. Management Science, 54, 1565-1578.

Hofstede, G., Hofstede, G. J., \& Minkov, M. (2010). Cultures and organizations: software of the mind ( $\left.3^{\mathrm{a}} \mathrm{Ed}.\right)$. NY: McGraw-Hill

Kirkpatrick, D. L. (1994). Evaluating training programs: The four levels. San Francisco, CA: Berrett-Koehler.

Kraiger, K. (2003). Perspectives on training and development. In W. C. Borman, D. R. Ilgen, \& R. J. Klimoski (Eds.). Handbook of Psychology. Vol. 12. Industrial and Organizational Psychology (pp. 171-192). Hoboken, NJ: Wiley.

Kraiger, K. (2008). Transforming our models of learning and development: Web-based instruction as enabler of thirdgeneration instruction. Industrial and Organizational
Psychology: Perspectives on Science and Practice, 1, 458-467.

Murphy, K. R. \& Myors, B. (1998). Statistical power analysis. A simple and general model for traditional and modern hypothesis tests. Mahwah, NJ: Erlbaum.

Noe, R. A. (2002). Employee training and development $\left(2^{\mathrm{a}}\right.$ ed.). NY: McGraw-Hill.

Patel, L. (2010). ASTD State of the industry report 2010. Alexandria, VA: American Society for Training and Development.

Salas, E., Diaz-Granados, D., Klein, C., Burke, C. S., Stagl, K. C., Goodwin, G. F., \& Halpin, S. M. (2008). Does team training improve team performance? A meta-analysis. Human Factors, 50, 903-933.

Salas, E. Nichols, D. R., \& Driskell, J. E. (2007). Testing three team training strategies in intact teams: A metaanalysis. Small Group Research, 39, 471-488.

Salas, E., Tannenbaum, S. I., Kraiger, K., \& Smith-Jentsch, K. A. (2012). The science of training and development in organizations: What matters in practice. Psychological Science in the Public Interest, 13, 74-101.

Salgado, J. F. (2007). La utilidad económica de la entrevista conductual estructurada en la selección de personal de la Administración Pública del País vasco. Revista de Psicología del Trabajo y de las Organizaciones, 23, 139154.

Scott, A., Gururajan, R., Moloney, C., \& Gururajan, V. (2006). A qualitative evaluation of the effectiveness of videoconferencing to support the training of orthopedic registrars. Journal of Telemedicine and Telecare, 12, 6970 .

Sitzmann, T., Krager, K., Stewart, D., \& Wisher, R. (2006). The comparative effectiveness of Web-based and classroom instruction: a meta-analysis. Personnel Psychology, $59,623-664$.
Manuscript received: 25/08/2012

Review received: 27/10/2012

Accepted: 30/10/2012 